\title{
Ecuaciones alométricas para estimar biomasa y carbono en Trichospermum mexicanum (DC.) Baill.
}

\author{
Allometric equations for estimating the above-ground biomass and carbon \\ in Trichospermum mexicanum (DC.) Baill.
}

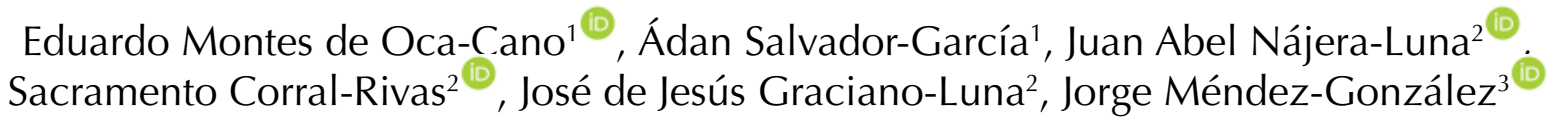

Recepción: 23 de enero de 2020

Aprobación: 21 de mayo de 2020

Montes de Oca-Cano, E., Salvador-García, A., Nájera-Luna, J. A., Corral-Rivas, S., Graciano-Luna, J. J., Méndez-González, J. (2020). Ecuaciones alométricas para estimar biomasa y carbono en Trichospermum mexicanum (DC.) Baill. Colombia Forestal, 23(2), 89-98.

\section{Resumen}

El presente estudio tuvo como objetivo ajustar ecuaciones lineales y no lineales para estimar carbono, por componente de hojas, ramas, fuste y total. Los resultados indican que, en el ámbito individual, el componente total muestra los mejores estadísticos de ajuste; mientras que las mayores variaciones se detectan en las ramas y en el fuste. Adicionalmente, se analizaron porcentajes de biomasa por componente donde se observa que el fuste aporta el $57 \%$ de la biomasa, las hojas el $9 \%$ y las ramas solamente el $34 \%$ de la biomasa total.

Palabras clave: ecuaciones alométricas, componentes vegetativos, bosque tropical.

\begin{abstract}
The aim of the present study was to adjust linear and non-linear equations in order to estimate the carbon component of leaves, branches and entire trees. The results indicate that at the individual level, the total component shows the best adjustment statistics, while major variation is detected in the branches and in the trunk. In addition, an analysis of the biomass percentages in each component indicates that the trunk accounts for $57 \%$ of the biomass, $9 \%$ for leaves and 34\%for branches.
\end{abstract}

Key words: allometric equations, vegetative components, tropical forest.

1 Instituto Tecnológico Superior de Zongolica. Carretera a la Compañía Col. Tepetitlanapa. C.P. 95005, Zongolica. Veracruz, México. montesdeoca.cano@hotmail.com. montesdeocaif@itszongolica.edu.mx., Autor para correspondencia.

2 Instituto Tecnológico de El Salto. El Salto, Durango, México. jalnajera@yahoo.com.mx, corral@gmail.com

3 Universidad Autónoma Agraria Antonio Narro, Coahuila, México., jorgemendezgonzalez@ gmail.com 


\section{INTRODUCCIÓN}

Las emisiones de gases de efecto invernadero (GEI) han y siguen afectando el clima del planeta Tierra de forma gradual; principalmente el bióxido de carbono $\left(\mathrm{CO}_{2}\right)$, el cual representa $76.7 \%$ del total de concentraciones de los GEI en la superficie de la atmósfera. Esto debido a que el ser humano quema exorbitantes cantidades de combustibles fósiles, como el petróleo, gas y carbón, además de la deforestación y otros agentes. Las consecuencias se presentan en el registro del incremento significativo de la temperatura global que causan, por ejemplo, el derretimiento de los polos y aumento del nivel del mar (IPCC, 2007).

La excesiva concentración atmosférica de los GEI, en especial la del bióxido de carbono, provoca manifestaciones como el calentamiento global, el cual se deriva del cambio climático. Estos fenómenos afectan directamente la integridad del planeta Tierra, ya que a mayor cantidad de GEI se aumenta la absorción de calor por parte de la superficie terrestre $y$, por ende, el planeta incrementará su temperatura global considerablemente (Secretaría del Medio Ambiente y Recursos Naturales, 2009).

Por fortuna, existe un medio natural de mitigar este gas. Los bosques son un elemento que permite reducir el dióxido de carbono en la atmósfera. Recordemos que, por medio de la fotosíntesis, las plantas y árboles capturan o asimilan el bióxido de carbono para incorporarlos a sus tejidos e incrementar su biomasa, para posteriormente liberar solo el oxígeno, lo que representa en términos ambientales un beneficio global. En este contexto, se debe fomentar el aprovechamiento sustentable de los bosques y asimismo preservar un ambiente para las generaciones futuras (Rojo et al., 2003).

No obstante, para poder concientizar a los seres humanos de cuán importante es la función específica de secuestro de carbono y el aprovechamiento sustentable de los ecosistemas forestales, es necesaria la intervención de un estudio adecuado para determinar con certeza la capacidad de almacenamiento de carbono, ya sea, de un ecosistema, un grupo de especies o una especie en particular (Gayoso, 2001). Además, mediante la inclusión de este tipo de estudios a proyectos de gestión para el pago de servicios ambientales (PSA), se pueden crear un conjunto de estrategias económicas viables financieramente que tomen en cuenta la vitalidad de los bosques y la necesidad de los dueños de los mismos (Burstein et al., 2002).

Estimar el contenido de carbono en la biomasa es la base para la gestión de PSA en la modalidad captura de carbono. Con base en este argumento se realizó un estudio de cuantificación de biomasa y estimación de carbono, en el ejido Gonzalo Vázquez Vela, del municipio de Tezonapa, del estado Veracruz de Ignacio de la Llave, México. Se analizaron 20 árboles de la especie Trichospermum mexicanum (DC.) Baill., utilizando el método destructivo para cuantificar biomasa aérea por componentes; para luego estimar el carbono almacenado, ajustar ecuaciones alométricas para estimar biomasa y carbono y, finalmente, estimar la fijación de bióxido de carbono en la biomasa en toneladas por hectárea (t.ha-1).

Analizar con cuanto contribuyen los árboles de Trichospermum mexicanum (DC.) Baill. a la mitigación de $\mathrm{CO}_{2}$ y a la regulación del cambio climático tendrá un impacto a medida que esta especie se mantenga en el proceso natural del carbono; como también al retribuir justa y redituablemente a los dueños de los bosques por su aporte a la conservación del medioambiente y a la reducción de la excesiva concentración de bióxido de carbono.

\section{MATERIALES Y MÉTODOS}

\section{Descripción del área de estudio}

La investigación se realizó en el ejido Gonzalo Vázquez Vela, perteneciente al municipio de Tezonapa, estado de Veracruz, México (18 $38^{\circ}$ '36.4" $\mathrm{N}$ y $96^{\circ} 48^{\prime} 34.9^{\prime \prime} \mathrm{O}$, y $850 \mathrm{~m}$ de altitud promedio); se localiza aproximadamente a $17 \mathrm{~km}$ de la 
cabecera municipal (Tezonapa) y a $5 \mathrm{~km}$ de la comunidad de Plan de Libres o Presidio. El ejido tiene un total de 1051 ha, de las cuales 500 están bajo un régimen de manejo forestal, con un permiso legal de aprovechamiento maderable. El clima, según García (1987), es cálido-húmedo, con una temperatura media anual mayor a $\operatorname{los} 22^{\circ} \mathrm{C}$, las precipitaciones en verano (promedio anual) son de $2000 \mathrm{~mm}$, el porcentaje de lluvia invernal es de $5 \%$, de un total anual de $10.2 \%$, y en los meses más secos la precipitación es mayor de 600 mm. La asociación de árboles la componen las especies: Alfaroa mexicana D.E. Stone., Trema micrantha (L.) Blume, Bumelia persimilis Hemsl., Licaria excelsa Kosterm., Quercus sp. Ocotea puberula (Rich.) Nees, Cordia alliodora (Ruiz \& Pav.), Cupania dentata DC. (Gutiérrez y Dorantes, 2004), y T. mexicanum (DC.) Baill. (Pennington y Sarukhán, 2005). Este lugar presenta altos índices de regeneración natural y crecimiento de T. mexicanum (árboles de 4, 7, 12 y 14 años de edad), en altitudes que oscilan entre los 800 y 900 m, con una distribución de $53 \%$ en 17 ha. Con base en lo anterior se eligió esta especie.

\section{Análisis destructivo}

Se utilizó el método de análisis destructivo y se siguió el procedimiento de Schlegel et al. (2000). Se seleccionaron 20 árboles representativos, sin enfermedades, ni deformidades y se distribuyeron en siete categorías diamétricas en intervalos de $5 \mathrm{~cm}$. Se registró el diámetro a la altura del pecho (DAP) y la altura total ( $\mathrm{H}$ en metros) de cada árbol, luego se derribaron, seccionaron y pesaron por componentes (fuste, ramas y hojas). El pesaje se realizó con una báscula romana de $200 \mathrm{~kg}$ de capacidad y $0.5 \mathrm{~kg}$ de precisión. Adicionalmente, se extrajeron muestras de cada componente: tres del fuste (rodajas $5 \mathrm{~cm}$ de espesor), dos muestras de ramas (de $10 \mathrm{~cm}$ de longitud) y dos bolsas de hojas (de 0.5 a $0.9 \mathrm{~kg}$ ), mismas que se pesaron de inmediato con un dinamómetro de $12 \mathrm{~kg}$ de capacidad y una precisión de $0.1 \mathrm{~kg}$.

\section{Cuantificación de biomasa aérea}

Las muestras de fuste, ramas y hojas se secaron a temperatura ambiente durante 45 días, hasta obtener el peso constante de cada muestra. Sin embargo, por la humedad relativa del ambiente (Acosta et al., 2011), algunas muestras (27 del fuste y 32 de las ramas) fueron trasladadas al Laboratorio de Semillas del Campo Experimental El Palmar, del Instituto Nacional de Investigaciones Forestales, Agrícolas y Pecuarias (Inifap). Las muestras se introdujeron en una estufa de secado convencional marca Riossa, a una temperatura de $100^{\circ} \mathrm{C}$ durante $48 \mathrm{~h}$; posteriormente, se registró su peso con una báscula analítica Excell SI1-32®, hasta conseguir el peso constante. Los pesos de las muestras restantes se corrigieron de acuerdo con el procedimiento descrito por Acosta et al. (2011). Con los datos de peso seco de las muestras, se procedió a calcular el peso seco total de biomasa por componente, por medio de la relación de peso seco de biomasa entre el peso fresco, y el resultante se multiplicó por el peso fresco correspondiente a cada componente (Acosta et al., 2002; Díaz et al., 2007).

\section{Contenido de carbono en la biomasa aérea}

El IPCC (2007) señala que generalmente el $50 \%$ del peso de biomasa seca es carbono, y argumenta que esta fracción puede ser usada para la conversión de biomasa a carbono. Sin embargo, por la variabilidad de porcentajes de carbono en la biomasa (Gayoso, 2001), en esta investigación se tomó como referencia la fracción 40.27 \% (Gayoso et al., 2002), con la finalidad de no sobre estimar y mantener el porcentaje de carbono.

\section{Ajuste de modelos alométricos para estimar biomasa y carbono}

Se ajustaron diez de los modelos alométricos que propusieron Gayoso et al. (2002) para estimar biomasa por componentes (fuste, ramas, hojas) y total. El ajuste de los modelos se realizó mediante 
el análisis de regresión con el paquete estadístico XLSTAT® versión 16.1.09.5739 (Addinsoft, 2014). Se utilizaron las variables: biomasa por componente y total (B en $\mathrm{kg}$ ), diámetro normal ( $\mathrm{D}$ en $\mathrm{cm}$ ) y altura total ( $\mathrm{H}$ en metros). Los modelos se evaluaron por los criterios estadísticos: coeficiente de determinación $\left(\mathrm{R}^{2}\right)$, error cuadrático medio (ECM), criterio de información Akaike (AIC), valor del estadístico $F(F)$ y la probabilidad de $F(P>F)$. Además, se consideró como criterio adicional la simplicidad del modelo (Montes de Oca et al., 2009).

\section{Fijación de bióxido de carbono equivalente en la biomasa aérea}

La fijación de $\mathrm{CO}_{2}$ e se estimó con el valor del contenido de carbono (componentes y total) y el coeficiente 3.67, que es el resultado de la relación del peso de la molécula de $\mathrm{CO}_{2}$ (44) entre el peso del átomo de C (12) (Pacheco et al., 2007; Rojo et al., 2003). Además, se estimó el $\mathrm{CO}_{2}$ e en $\mathrm{Mg} \cdot \mathrm{ha}^{-1}$ : se utilizó el promedio del diámetro normal, el promedio de biomasa aérea total $(\mathrm{kg})$, el promedio de contenido de carbono total $(\mathrm{kg})$ y el promedio total de $\mathrm{CO}_{2}$ e (kg) fijado en la biomasa aérea. El número de árboles. ha $^{-1}$ se determinó con base en la información de los puntos de análisis destructivo (20 sitios de $1000 \mathrm{~m}^{2}$ distribuidos en las $17 \mathrm{ha}$ ), cubriendo el mayor gradiente de árboles representativos de esta especie.

\section{RESULTADOS}

\section{Biomasa por componente y total}

El fuste presentó un intervalo de 24.96 a $479.46 \mathrm{~kg}$, las ramas de 14.25 a $270.42 \mathrm{~kg}$, mientas que en las hojas fue de 7.72 a $58.44 \mathrm{~kg}$. Estos valores indican que el mayor porcentaje de biomasa se encuentra en el fuste (57\%), en rangos de 41.62 a $69.69 \%$; seguido del componente ramas (34\%), con un rango desde 21.52 hasta $44.34 \%$; y, finalmente, el componente hojas (9\%), con un rango de 6.00 a $16.45 \%$.

En el caso del porcentaje de humedad en promedio, el orden fue: 52.75, 38.32, 36.40 \%, para fuste, ramas y hojas, respectivamente, con un promedio para el total de $45.20 \%$. El peso verde de los 20 árboles de T. mexicanum fue de $16.65 \mathrm{Mg}$, mientras que el peso seco la biomasa total fue de $7.79 \mathrm{Mg}$.

La suma de biomasa de cada componente correspondió a la biomasa aérea total de cada árbol (tabla 3).

\section{Carbono por componente y total}

El $58 \%$ de carbono aéreo se encuentra en el fuste, en un rango de 10.05 a 193.08 kg; siguiendo las ramas con $34 \%$, en un rango de 5.74 a $108.90 \mathrm{~kg}$; y, por último, las hojas con $8 \%$, en un rango de 3.11 a $23.54 \mathrm{~kg}$. Los valores de carbono total oscilaron en un rango de 18.91 a $325.51 \mathrm{~kg}$ (tabla 1).

\section{Ecuaciones para estimar biomasa y carbono aéreos}

Los criterios estadísticos de ajuste determinaron al modelo 4 (fuste, ramas y total) y 6 (hojas) como los mejores para estimar la biomasa y carbono en $T$. mexicanum (tabla 2). En todos los casos los valores estadísticos de $\mathrm{F}$ fueron altos; además, la probabilidad fue inferior $(P<0.0001)$, con un riesgo de menos $0.01 \%$. La ponderación de $\mathrm{R}^{2}$ de los modelos fue de un 0.88 , lo que indica que el $88 \%$ de la variabilidad de biomasa aérea es explicada por el diámetro medio (D) y por la altura total del árbol (H) en su caso (Addinsoft, 2014). El modelo 4 fue el mejor para estimar la biomasa aérea, y se comprobó que el uso exclusivo del diámetro medio (D) provee de considerable información al modelo para la predicción. En contraste, el modelo 6 estimó eficientemente la biomasa en las hojas, esto obedece a factores fotosintéticos (competencia por la luz y el $\mathrm{CO}_{2}$ ), y se refleja en la producción foliar, 
Tabla 1. Características dasométricas, valores de biomasa y carbono en árboles de T. mexicanum (DC.) Baill.

\begin{tabular}{ccccccc}
\hline Árbol & $\begin{array}{c}\text { Categoría } \\
\text { diametrica }\end{array}$ & CD & D & H & BT & CT \\
\hline 1 & 10 & $10-15$ & 13.3 & 10.6 & 46.94 & 18.91 \\
2 & 15 & $15-20$ & 16.8 & 12.5 & 77.91 & 31.38 \\
3 & 15 & $15-20$ & 17.5 & 10.8 & 104.93 & 42.26 \\
4 & 20 & $20-25$ & 24.1 & 13.2 & 232.01 & 93.43 \\
5 & 20 & $20-25$ & 24.5 & 15.5 & 212.65 & 85.64 \\
6 & 20 & $20-25$ & 24.8 & 14.3 & 186.75 & 75.20 \\
7 & 20 & $25-30$ & 26.1 & 14.7 & 352.78 & 142.06 \\
8 & 25 & $25-30$ & 30.3 & 13.9 & 413.86 & 166.66 \\
9 & $25-30$ & 30.7 & 17.2 & 378.40 & 152.38 \\
10 & 25 & $25-30$ & 30.8 & 15.8 & 350.65 & 141.21 \\
11 & 30 & $30-35$ & 31.2 & 14.8 & 334.16 & 134.57 \\
12 & 30 & $30-35$ & 32.1 & 14.5 & 482.75 & 194.41 \\
13 & 30 & $30-35$ & 32.4 & 13.8 & 491.91 & 198.09 \\
14 & 30 & $30-35$ & 32.4 & 15.9 & 529.70 & 213.31 \\
15 & 30 & $30-35$ & 34.3 & 15.1 & 455.40 & 183.39 \\
16 & 35 & $35-40$ & 37.5 & 18.7 & 502.30 & 202.28 \\
17 & 35 & $35-40$ & 37.5 & 18.1 & 489.90 & 197.28 \\
18 & 35 & $35-40$ & 39.1 & 18.1 & 656.24 & 264.27 \\
19 & 40 & $40-45$ & 41 & 19.4 & 685.29 & 275.97 \\
20 & 40 & $40-45$ & 41.5 & 20.7 & 808.33 & 325.51 \\
Media & & & 29.8 & 15.3 & 389.65 & 156.91 \\
\hline
\end{tabular}

CD: categoría diamétrica (cm); D: diámetro normal $(\mathrm{cm})$; H: altura total $(\mathrm{m})$; BT: biomasa total $(\mathrm{kg})$; $\mathrm{CT}$ : carbono total $(\mathrm{kg})$. El contenido de $\mathrm{C}$ en los 20 árboles de T. mexicanum representó $3.13 \mathrm{Mg}$, cifra que corresponde al 40 \% de la biomasa.

Tabla 2. Ecuaciones alométricas generadas para estimar biomasa y carbono por componente y total en T. mexicanum (DC.) Baill.

\begin{tabular}{ccccccc}
\hline EI & $\mathbf{C p}$ & $\mathbf{M}$ & Ecuación & $\mathbf{R}^{\mathbf{2}}$ & ECM & AIC \\
\hline \multirow{4}{*}{ B } & $\mathrm{F}$ & 4 & $\mathrm{Y}=-21.262+0.265\left(\mathrm{D}^{2}\right)$ & 0.86 & 47.54 & 148.63 \\
& $\mathrm{R}$ & 4 & $\mathrm{Y}=-16.874+0.156\left(\mathrm{D}^{2}\right)$ & 0.86 & 29.04 & 129.90 \\
& $\mathrm{~T}$ & 4 & $\mathrm{Y}=-33.565+0.449\left(\mathrm{D}^{2}\right)$ & 0.93 & 55.18 & 154.29 \\
& $\mathrm{H}$ & 6 & $\mathrm{Y}=-6.702+1.003(\mathrm{D})+0.0004\left(\mathrm{D}^{2} \mathrm{H}\right)$ & 0.88 & 4.84 & 62.67 \\
& $\mathrm{~F}$ & 4 & $\mathrm{Y}=-8.562+0.106\left(\mathrm{D}^{2}\right)$ & 0.86 & 19.14 & 114.06 \\
& $\mathrm{R}$ & 4 & $\mathrm{Y}=-6.794+0.062\left(\mathrm{D}^{2}\right)$ & 0.86 & 11.69 & 95.34 \\
$\mathbf{C}$ & $\mathrm{T}$ & 4 & $\mathrm{Y}=-13.516+0.180\left(\mathrm{D}^{2}\right)$ & 0.93 & 22.22 & 119.72 \\
& $\mathrm{H}$ & 6 & $\mathrm{Y}=-2.698+0.403(\mathrm{D})+0.0001\left(\mathrm{D}^{2} \mathrm{H}\right)$ & 0.88 & 1.95 & 28.10 \\
\hline
\end{tabular}

El: elemento (B: biomasa; C: carbono); Cp: componente (F: fuste; R: ramas; H: hojas; T: total); M: número del modelo;

$\mathrm{R}^{2}$ : coeficiente de determinación; ECM: error cuadrático medio; AIC: criterio de información Akaike.

que incrementa directamente con relación a la altura total del árbol (H) (Picard et al., 2012).

El comportamiento de biomasa estimada fue: para el fuste valores de 29.18 a $422.32 \mathrm{~kg}$, mientras que las ramas oscilaron desde 10.65 hasta
$252.34 \mathrm{~kg}$, y las hojas tuvieron un valor de 7.39 a $47.50 \mathrm{~kg}$. Para la biomasa total los valores fueron: 49.70 a $725.79 \mathrm{~kg}$, con un promedio de $389.60 \mathrm{~kg}$. El promedio de la diferencia en cada componente (contra la biomasa del análisis destructivo) fue 
mínimo $(37.14,18.07,3.69 \mathrm{~kg}$, para fuste, ramas y hojas, respectivamente); para la biomasa total se presentó un rango de 2.75 a $82.54 \mathrm{~kg}$, y un promedio de $44.32 \mathrm{~kg}$. Los resultados indican que la biomasa aérea de T. mexicanum se predijo eficientemente, ya que los valores de diferencia no fueron de gran magnitud (figura 1).

El carbono por componentes fue: para el fuste valores de 10.29 a $175.03 \mathrm{~kg}$, mientras que las ramas presentaron valores de 4.31 a $101.36 \mathrm{~kg}$, y para las hojas de 2.86 a $17.63 \mathrm{~kg}$. La estimación del contenido de carbono total presentó valores de 18.43 a 297.52 kg, y un promedio de 158.74 kg. El promedio de la diferencia por componentes (contra el resultado del factor de conversión de carbono) no tuvo gran magnitud, para el carbono total fue de tan solo $17.90 \mathrm{~kg}$. La tendencia de los valores de carbono total con respecto al diámetro medio (D), muestran la linealidad del modelo, y se comprobó la hipótesis de que el uso del diámetro medio (D) origina predicciones de biomasa y carbón (B C) eficientes (figura 1).

\section{Bióxido de carbono equivalente en la biomasa aérea}

El $\mathrm{CO}_{2}$ por árbol tuvo un rango de 69.32 a 1193.55 $\mathrm{kg}$, y un promedio de $575.33 \mathrm{~kg}$. El fuste representó la mayor proporción de $\mathrm{CO}_{2} \mathrm{e}$ en un rango de 36.87 a $707.96 \mathrm{~kg}$, las ramas presentaron un rango de 21.05 a $399.30 \mathrm{~kg}$, y las hojas presentaron un rango de 11.40 a $86.30 \mathrm{~kg}$. También se obtuvo la acumulación de biomasa, carbono y $\mathrm{CO}_{2}$ e en Mg.ha-1 (tabla 3).

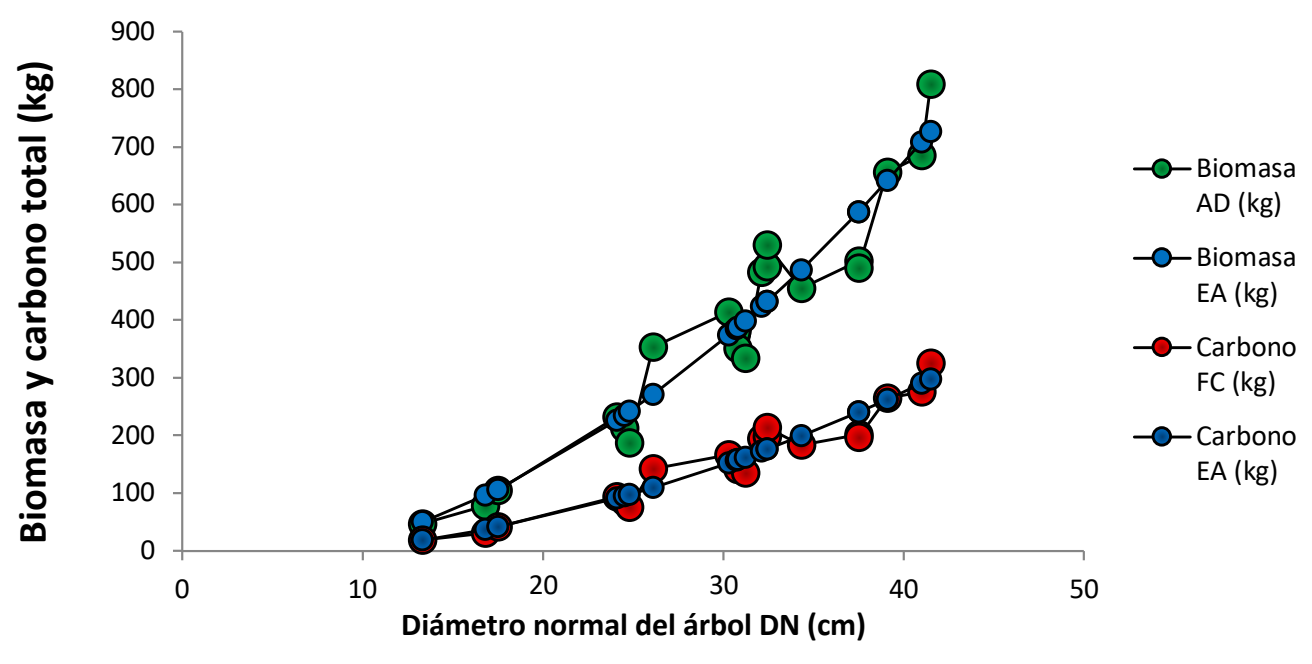

Figura 1. Valores de biomasa y carbono total de Trichospermum mexicanum (DC.) Baill. (AD: análisis destructivo; FC: factor de conversión de carbono; EA: ecuaciones alométricas).

Tabla 3. Características dasométricas, biomasa, carbono y bióxido de carbono equivalente de T. mexicanum (DC.) Baill.

\begin{tabular}{ccccccccc}
\hline Árb. & $\mathbf{D}$ & $\mathbf{H}$ & \multicolumn{2}{c}{$\mathbf{B}$} & \multicolumn{2}{c}{$\mathbf{C}$} & \multicolumn{2}{c}{$\mathbf{C O}_{\mathbf{2}} \mathbf{e}$} \\
\hline$\left(\mathrm{ha}^{-1}\right)$ & $(\mathrm{cm})$ & $(\mathrm{m})$ & $(\mathrm{kg})$ & $\left(\mathrm{Mg} \mathrm{ha}^{-1}\right)$ & $(\mathrm{kg})$ & $\left(\mathrm{Mg} \mathrm{ha}^{-1}\right)$ & $(\mathrm{kg})$ & $\left(\mathrm{Mg} \mathrm{ha}^{-1}\right)$ \\
200 & 29.8 & 15.3 & 389.65 & 77.92 & 156.91 & 31.38 & 575.33 & 115.06 \\
\hline
\end{tabular}

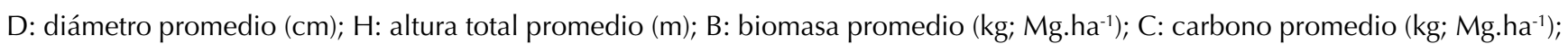
$\mathrm{CO}_{2} \mathrm{e}$ : bióxido de carbono equivalente $\left(\mathrm{kg} ; \mathrm{Mg} \cdot \mathrm{ha}^{-1}\right)$. 


\section{DISCUSIÓN}

\section{Biomasa por componente y total}

Con respecto al mayor porcentaje de biomasa promedio, Acosta et al. (2011) confirmaron que el fuste de un árbol (Clethra mexicana) presenta la mayor proporción (fuste: $45.15 \%$; ramas: 36.24 \%; hojas: $18.61 \%) ;$ además, reportan porcentajes de humedad más altos (Clethra mexicana: $61.17 \%$; Alnus arguta: $52.58 \%$ ).

El aumento de biomasa promedio es directamente proporcional al incremento del diámetro promedio. Gómez et al. (2011) coinciden con lo anterior, pues analizaron 12 árboles de Quercus magnoliaefolia de los cuales 6 presentaron un diámetro promedio de 10.1 a $31.5 \mathrm{~cm}$, y la proporción de biomasa promedio fue de 40.4 a $863.7 \mathrm{~kg}$.

\section{Carbono por componente y total}

Los valores de carbono promedio se estimaron de la forma indirecta; este procedimiento ha sido utilizado por algunos autores (Méndez et al., 2011; Douterlungne et al., 2013). Montes de Oca et al. (2012) afirman que de esta forma se obtiene un resultado conservador. En términos de concentración (\% C) la variación es de 34.86 a 48.31 \%, y se presenta en cada especie y en cada tejido vegetal de un árbol; también se han presentado variaciones menores, desde 41.05 hasta $46.65 \%$ (Gayoso, 2001). Usar un factor de conversión para la evaluación del contenido de carbono promedio en la biomasa promedio.

\section{Ecuaciones para estimar biomasa y carbono aéreos}

Montes de Oca et al. (2009) ajustaron 13 modelos, incluyendo los 10 de este estudio, en árboles de Pinus durangensis. Los mejores fueron el modelo 1 (ramas y hojas), 13 (fuste) y 3 (total) ( $R^{2}: 0.74$, 0.86 y 0.86 respectivamente); estas diferencias se presentaron principalmente por el uso del diámetro en la base (DB), y a que la altura de los árboles fue menor a dos metros, y generalmente los modelos usan el diámetro a 1.30 m. Por otro lado, Méndez et al. (2011) ajustaron nueve de los modelos para estimar la Biomasa en el fuste y total, en una plantación de Pinus devoniana y Pinus pseudostrobus, y el modelo $4\left(Y=a+b D^{2}\right)$ fue uno de los mejores; sin embargo, reportó valores más bajos de $\mathrm{R}^{2}$ 0.85 y $0.84 ; 0.90$ y 0.85 , respectivamente.

Algunos autores (Ter-Mikaelian y Korzukhin, 1997; Acosta et al., 2002; Díaz et al., 2007) han usado la relación $Y=b X^{k}$ en su forma normal $y$ lineal, y comprobaron que es útil para estimar la biomasa aérea. Chave et al. (2005) procedieron a calibrar (2410 observaciones) un modelo generalizado que permite amplitud de predicción, abarcando muchas especies y varias zonas ecológicas, desde las zonas más secas, hasta las más húmedas. Sin embargo, Návar et al. (2013) mencionan que un modelo general produce resultados estadísticos de ajuste muy bajos, y concluyen que se debe tener cuidado para elegir el modelo y desarrollar una ecuación propia.

Se comprobó que el análisis destructivo de biomasa aérea es determinante para aplicar alometría a nivel árbol y especie (Schlegel et al., 2000; Návar et al., 2013).

También se comprobó que el uso exclusivo de la ecuación 4 estimó la biomasa y carbono total del árbol, por tanto, no es necesario evaluar cada componente para determinar la biomasa y carbono aéreos (Acosta et al., 2011). En contraste, la ecuación 6 solo estimó la biomasa en hojas; esta puede ser usada si se desea determinar la producción foliar.

\section{Bióxido de carbono equivalente en la biomasa aérea}

Méndez etal. (2011) estimaron la biomasa, carbono y $\mathrm{CO}_{2}$ e de una plantación mixta de Pinus devoniana y Pinus pseudostrobus (564 árboles.ha-1), y a los 
13 años de edad acumuló 39.3 Mg B.ha' ${ }^{-1}, 19.6 \mathrm{Mg}$ C.ha ${ }^{-1}$ y $72.0 \mathrm{Mg} \mathrm{CO}_{2}$ ha $^{-1}$ para ambas especies. Las diferencias son menores, debido a la edad, el D $(18.5 \mathrm{~cm})$ y la $\mathrm{H}(8.9 \mathrm{~m})$, además del desarrollo ontogénico de cada especie (Picard et al., 2012). Por otro lado, Douterlungne et al. (2013) estimaron la acumulación de carbono en varias especies, entre ellas T. mexicanum, y su acumulación en sus dos primeros años fue de $11.9 \mathrm{Mg} \mathrm{C}$.ha $^{-1}$ (D: 0 a 39.8 cm; H: 0.50 a $19 \mathrm{~m}$ ), cifra que difiere (si se extrapolara) de la de este estudio (31.38 Mg C.ha-1). Sin embargo, este autor menciona que esta tasa de acumulación no es extrapolable para cuantificar el carbono a largo plazo, debido a que la tasa decae o aumenta por factores como deforestación y competencia, y aún si se extrapolará a las edades de este estudio (4, 7, 12 y 14 años), la tasa de acumulación es mucho menor.

Ordóñez (1999) afirma que las selvas presentan hasta $120 \mathrm{Mg}$ carbono en la biomasa aérea; esta cifra es considerable ya que las selvas contienen gran cantidad de especies, además deben tener en cuenta los componentes suelo, arbustos y biomasa en descomposición. En términos acumulativos de carbono en la biomasa aérea, la tasa en $\mathrm{Mg}$.ha ${ }^{-1}$ puede ser subestimada, y puede ser de más magnitud; para no crear incertidumbre, es necesario realizar más investigaciones con respecto la evaluación de captura de carbono en los bosques; utilizar la combinación de métodos directos e indirectos para una mayor precisión y, según sea el caso, actualizarlos o modificarlos, ya que serán fundamentales para estimaciones futuras.

\section{CONCLUSIONES}

El método de análisis destructivo en la biomasa aérea fue determinante para evaluar la captura de carbono en los árboles de Trichospermum mexicanum. La ecuación alométrica que incluyó solo al diámetro, estima eficientemente la biomasa y carbono aéreo. La ecuación que incluyó la combinación de diámetro y altura, solo estimó la biomasa y carbono en el componente hojas.

Las estimaciones de biomasa y carbono son confiables, por tanto, el procedimiento en este estudio puede ser usado en otras especies en condiciones de desarrollo similares. Es recomendable ajustar todos los modelos para determinar cuál o cuáles se integran a las necesidades de cada condición.

La evaluación de carbono en árboles de Trichospermum mexicanum generó un resultado que permite conocer con cuánto se contribuye para mitigar la excesiva concentración del bióxido de carbono en la atmósfera, así como apoyar en el mantenimiento del equilibrio del sistema climático.

\section{AGRADECIMIENTOS}

A las personas del Núcleo Ejidal Gonzalo Vázquez Vela, Tezonapa, Veracruz, por aceptar la realización de esta investigación en la superficie de sus parcelas, pero sobre todo por su confianza y por brindarnos todas las facilidades. Al Instituto Tecnológico Superior de Zongolica (ITSZ). AI Campo Experimental El Palmar, del Instituto Nacional de Investigaciones Forestales, Agrícolas y Pecuarias (Inifap).

\section{CONFLICTO DE INTERESES}

Los autores declaran no tener conflicto de intereses.

\section{CONTRIBUCIÓN POR AUTOR}

E. M. de O.C. ideó la investigación y redacción del manuscrito; A.S.G. trabajó en la investigación con los trabajos en campo; J.A.N.L., S.C.R. y J.G.L. analizaron estadísticamente los datos de la investigación y J. M.G. revisó la redacción del manuscrito. 


\section{REFERENCIAS}

Acosta, M., Carrillo, A. y Gómez, V. (2011). Estimación de biomasa y carbono en dos especies de bosque mesófilo de montaña. Revista mexicana de Ciencias Agrícolas, 2(4), 529-543.

https://doi.org/10.29312/remexca.v2i4.1638

Acosta, M., Vargas, A., Velázquez, M. y Etchevers, B. (2002). Estimación de la biomasa aérea mediante el uso de relaciones alométricas en seis especies arbóreas en Oaxaca, México. Agrociencia, 36(6), 725-736.

Addinsoft Inc. (2014). User's guide, version 16.1.09.5739. XLSTAT 2014. Nueva York.

http://www.xlstat.com/es/

Brown, S. (1997). Estimating biomass and biomass change of tropical forests: a Primer. FAO (Food and Agriculture Organization). Forestry Paper, 134. Roma.

Brown, R., Gillespie, A. y Lugo, E. (1989). Biomass estimation methods for tropical forests with applications to forest inventory data. Forest. Sciencie, 35(4), 881-902.

Burstein, J., Chapela y M., G., Aguilar, J., y de León, E. (2002). Informe sobre la propuesta de pago por servicios ambientales en México. Pago por Servicios Ambientales en Las Américas. México.

Chave, J. (2005). Tree allometry and improved estimation of carbon stocks and balance in tropical forests. Oecologia, 145(1), 87-99.

https://doi.org/10.1007/s00442-005-0100-x

Díaz, F., Acosta, M., Carrillo, E., Buendía, R., Flores, A. y Etchevers, B. (2007). Determinación de ecuaciones alométricas para estimar biomasa y carbono en Pinus patula Schl. et Cham. Madera y Bosques, 13(1), 25-34.

https://doi.org/10.21829/myb.2007.1311233

Douterlungne, D., Herrera, G., Ferguson, I. y Soto, P. (2013). Ecuaciones alométricas para estimar biomasa y carbono de cuatro especies leñosas neotropicales con potencial para la restauración. Agrociencia, 47, 385-397.

García, E. (1987). Modificaciones al Sistema de Clasificación Climática de Köppen. 4a. ed. México.
Gayoso, J. y Alarcón, D. (2002). Contenido de carbono y funciones de biomasa en especies nativas y exóticas. Valdivia: Proyecto Fondef.

Gayoso, J. (2001). Medición de la capacidad de captura de carbono en bosques nativos y plantaciones de Chile. En Taller Secuestro de Carbono (pp. 22). Universidad Austral de Chile.

Gómez, D., Etchevers, B., Monterrosos, A. y Tinoco, R. (2011). Ecuaciones alométricas para estimar biomasa y carbono en Quercus magnoliaefolia Née. Revista Chapingo Serie Ciencias Forestales y del Ambiente, 17(2), 261-272.

https://doi.org/10.5154/r.rchscfa.2010.11.117

Gutiérrez, C. y Dorantes, L. (2004). Especies forestales de uso tradicional del estado de Veracruz. Veracruz: Conafor, Conacyt y UV.

Intergovernmental Panel on Climate Change (2007). Cambio climático 2007: Informe de síntesis. En R. K. Pachauri y A. Reisinger (eds.), Contribución de los grupos de trabajo I, II y III al cuarto informe de evaluación del grupo intergubernamental de expertos sobre el cambio climático. Ginebra, Suiza.

Microsoft (2020). EXCELL SI1-32® (versión windows 7). https://support.office.com/es-emx

Méndez, G., Luckie, N., Capó, y Nájera, L. (2011). Ecuaciones alométricas y estimación de incrementos en biomasa aérea y carbono en una plantación mixta de Pinus devoniana Lindl. y Pinus pseudostrobus Lindl., en Guanajuato, México. Agrociencia, 45(4), 479-491.

Montes de Oca, E., Rojas, R., Nájera, L., Méndez, G. y Graciano, L. (2012). Estimación de carbono almacenado en la regeneración natural de Pinus durangensis Martínez en El Salto, Durango. Colombia Forestal, 15(2), 151-159.

https://doi.org/10.14483/udistrital.jour.colomb. for.2012.2.a01

Montes de Oca, E., García, P., Nájera, L. y Méndez, G. (2009). Ajuste de ecuaciones de biomasa para Pinus durangensis (Martínez M.) en la región de El Salto, Durango. Revista Chapingo Serie Ciencias Forestales y del Ambiente, 15(1), 65-71.

Návar, C. Rodríguez, F. y Domínguez, C. (2013). Ecuaciones alométricas para árboles tropicales: 
aplicación al inventario forestal de Sinaloa, México. Agronomía Mesoamericana, 24(2), 347-356. https://doi.org/10.15517/am.v24i2.12534

Ordóñez, D. (1999). Captura de carbono en un bosque templado: El caso de San Juan Nuevo, Michoacán. México: Instituto Nacional de Ecología; Semarnap.

Pacheco, A., Aldrete, A., Gómez, G. Fierros, G. y Vaquera, H. (2007). Almacenamiento de carbono en la biomasa aérea de una plantación joven de Pinus greggii Engelm. Revista Fitotecnia mexicana, 30(3), 251-254.

Pennington, T. y Sarukhán, J. (2005). Arboles tropicales de México: manual para la identificación de las principales especies (3era ed.). México: Universidad Nacional Autónoma de México; Fondo de Cultura Económica.

Picard, S. y Henry, M. (2012). Manual de construcción de ecuaciones alométricas para estimar el volumen y la biomasa de los árboles: del trabajo de campo a la predicción. Roma, Italia: Organización de las
Naciones Unidas para la Alimentación y la Agricultura; Centre de coopération Internationale en Recherche Agronomique pour le Développement.

Rojo, M. y Velázquez, M. (2003). Las masas forestales como sumideros de $\mathrm{CO}_{2}$ ante un cambio climático global. Revista Chapingo Serie Ciencias Forestales y del Ambiente, 9(1), 57-67.

Secretaria de Medio Ambiente y Recursos Naturales (2009). Serie ¿Y el medio ambiente? Cambio climático: ciencia, evidencia y acciones. México: Dirección General de Estadística e Información Ambiental.

Schlegel, B., Gayoso, J. y Guerra, J. (2000). Manual de procedimientos: muestreos de biomasa Forestal. Valdivia, Chile: Proyecto Fondef; Universidad Austral de Chile.

Ter-Mikaelian, T. y Korzukhin, M. (1997). Biomass equation for sixty-five north American tree species. Forest Ecology and Management, 97(1), 1-24. https://doi.org/10.1016/S0378-1127(97)00019-4

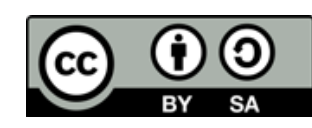

Colombia Forestal •ISSN 0120-0739 • e-ISSN 2256-201X • Bogotá-Colombia • Vol. 23 No. 2 • Julio-diciembre de $2020 \bullet$ pp. 89-98. 